\title{
Metaphorical Reasoning With an ECONOMical Set OF MapPINGS
}

(Raciocínio Metafórico com um Conjunto Econômico de Mapeamentos)

\author{
A.M. Wallington, J.A. Barnden, S.R. Glasbey, M.G. LeE* \\ (School of Computer Science, University of Birmingham, \\ Edgbaston, Birmingham B15 2TT, United Kingdom)
}

\begin{abstract}
This paper discusses the nature of the metaphorical transfer from the source domain to the target domain. More specifically, it explores the question as to how the mapping links between features of the source and the target are created. It is argued that, for many metaphors, it is incorrect to assume that all the elements of the source domain map to elements of the target domain, and that a much more economical set of mappings should be used instead.
\end{abstract}

KEY-WORDS: Metaphorical transfer, mappings, source and target domains

RESUMO: Este trabalho discute a natureza da transferencia metafórica entre domínio fonte e domínio alvo. Argumenta-se que, para grande parte das metáforas, não seria correto afirmar que todos os elementos do domínio alvo sejam mapeados para os elementos do dominio fonte, e que um conjunto bem mais econômico de mapeamentos é utilizado. PALAVRAS-CHAVE: transferência metafórica, domínios conceptuais, mapeamentos

\section{Introduction}

In this paper, we shall take the common view that metaphor involves some notion of features, relations, propositions, etc being transferred from

\footnotetext{
* This paper is based on a presentation given at the Conference on Metaphor and Thought, Pontifical Catholic University of São Paulo, Brazil, 21-25 October 2002. We would like to thank the conference participants for their helpful comments, criticisms and suggestions. The research was supported by EPSRC grant GR/M64208.
} 
the source or vehicle domain to the target or tenor domain and that as a result, the metaphorical utterance will produce information about the target domain, insight into it, or the highlighting of parts of it. We shall also assume that the transfer is from the source to the target in that the source is predominantly used to illuminate the target and not the other way around, (but cf.: Barnden et al 2004). Most importantly for the purposes of this paper, we shall assume that the transfer utilises mapping links between features etc of the source and the target. And this raises the question as to how the links are created? One possibility is that since metaphor is similar in many respects to analogy (see, for example, Gentner 1983), then these links arise via analogy, that is via the underlying conceptual similarity between the source and target domains. For example, a relation, $R$, between $\mathrm{A}$ and $\mathrm{B}$ in the source domain is analogous to the relation $\mathrm{R}$ between $\mathrm{P}$ and $\mathrm{Q}$ in the target domain, with $\mathrm{A}$ mapping onto $\mathrm{P}$ and $\mathrm{B}$ mapping onto $\mathrm{Q}$. However, considering this approach from a computational perspective shows up a problem; it places a great burden on what is meant by similarity and how it is recognised. We can perhaps assume that both the target domain and the source domain can be structured in terms of a set of objects and the relations between these objects. Indeed, the situation is likely to be more complicated still, with higher-order relations between objects that themselves express a relation between objects. However, in order to uncover which relations and which objects in the source map optimally to which objects and which relations in the target, we have little option but to systematically try out all possible mappings. It should not be hard to appreciate that this can be an extremely laboured process. ${ }^{1}$

Of course, it may be that metaphor understanding is an extremely laboured process when compared with the understanding of literal language, but the psycholinguistic evidence suggests otherwise (cf.: Ortony et al 1987). Perhaps more importantly, the search for structural similarity as a basis for the mapping links and transfer ignores the possibility that whatever might once have been the case, either in the history of the language or in the personal history of the individual learning the language, many source to target links already form a conventional part of a person's conceptual system

\footnotetext{
1 Veale and Keane (1997), show that the problem of determining the largest common isomorphic pair of sub-graphs shared by two semantic structures called the Source and Target, such that a systematic and coherent 1-to-1 mapping of elements from the Source to the Target is established, is NP-Hard, cf., also Indurkyha (1987) and Falkenhainer et al., (1989).
} 
and do not need to be created anew whenever a metaphor is uttered or understood. Conceptual metaphors, such as LOVE-IS-A-JOURNEY or MINDIS-PHYSICAL-SPACE, which take the form of a collection of systematically related correspondences or links, possess a vast generative capacity, allowing the underlying metaphor to manifest itself in a wide range of surface forms. (cf.: Lakoff \& Johnson 1987).

But if there exist some pre-existing source to target mappings, does this mean that all the features, relations, propositions, etc from the source domain that are needed for understanding the utterance are transferred to the target domain via pre-existing mapping links? And, if not, how, if at all, is the informational contribution of these other source domain features determined? Are they transferred to the target by mapping links that are similar to the established links but made on the fly, or are they used in some other manner? To explore these issues, let us take some wellestablished conceptual metaphors and check whether the mapping links adequately cover the range of entities contained in metaphorical utterances in mundane discourse that manifest these conceptual metaphors.

\section{Map-Transcending Elements and Inferencing}

\subsection{Inferencing}

Lakoff (1993: p. 210) gives the following example of a metaphorical song lyric:

We're driving in the fast lane on the freeway of love.

The example is argued to be a use of the familiar conceptual metaphor LOVE IS A JOURNEY in which the progress of the love relationship is viewed as a journey, the lovers are viewed as people undertaking the journey, the love relationship as the vehicle in which they are travelling, and difficulties in the relationship correspond to impediments to travel (p. 207). These correspondences do not include mappings for the notions of driving, freeway or fast lane. Consequently, if there are no mappings for these notions, then it would appear that the specified mapping links do not always adequately cover the range of entities contained in metaphorical utterances in mundane discourse, although somewhat confusingly, Lakoff remarks (p. 211) that the knowledge structures associated with the words freeway and fast lane are mapped. 
However, whether there are or there are not sufficient pre-existing mapping links to cope with this particular example, in general it would seem inconceivable that a reader ${ }^{2}$ would possess a mapping for more than a minority of the notions in the source domain of any given metaphorical utterance. So, should we assume that the reader performs an on-the-fly search for correspondences for any unmapped elements, henceforth 'maptranscending elements' or 'MTEs', perhaps along the lines discussed in the Introduction, by searching for relations between MTEs and entities for which there is already an existing mapping? We would not want to say that this is never done, given the fact that analogical reasoning exists. However, given the likely computational cost involved we shall assume that this is to be avoided whenever possible. In fact we shall enshrine it as a principle:

Map-Extension Minimization.

By default, an extension of the mapping(s) in the stored conceptual metaphor(s) used in an utterance, to cover map-transcending aspects of the utterance, should not be sought. It should only be sought when there is a specific need for it posed by the discourse understanding task.

So, what alternatives are there to creating map extensions? In the 1993 article referred to earlier, Lakoff states that the reader can reason, within the terms of the source domain, that the journey alluded to in the utterance is exciting, using the source-domain knowledge that driving in the fast lane of a freeway is exciting. The excitement in the source domain is for the travellers. This maps, apparently, to excitement for the lovers, in the target domain. Consequently, the map-transcending elements: 'fast lane', 'freeway', and 'driving', do not partake in any of the actual mappings of the LOVE IS A JOURNEY conceptual metaphor, but rather they are used in a process of reasoning to infer something that is mapped, namely the emotion of excitement.

Note that we are not merely saying that a process of inference is involved in metaphor comprehension; a widely accepted view. It is worth contrasting the above approach with another approach involving the making of inferences and which also appears to be endorsed in Lakoff s 1993 article (pp. 207-8). In a description of the inferences involved in

2 We will use the term 'reader' to refer to the person who understands the metaphor, regardless of whether the vehicle is written or spoken. 
understanding the metaphorical use of the phrase "we're stuck", each step in the reasoning process made in the source domain finds its counterpart in the target domain. Thus, the inference pattern as a whole is mapped from one domain to another.

[It] "is an example of an inference pattern that is mapped from one domain to another." (Lakoff 1993:208)

By contrast, the approach sketched above just involves the transfer of the conclusion; there is no assumption that each step in the process finds a target domain counterpart.

Another point to make is that the conclusion, which here consists of the invariant mapping from 'excitement' in the source to 'excitement' in the target, is not usually specified as forming a part of LOVE IS A JOURNEY conceptual metaphor. Indeed, it would be surprising if such a mapping were specified as being part of this particular conceptual metaphor since we would claim that it, or a mapping involving some other emotion, would appear to be an option for all conceptual metaphors.

If this 'inferencing' approach can be sustained and made more explicit, then we have a start of a solution to the problem of elements in utterances that do not have a conventional source to target link. The reader will make use of her knowledge about the source domain plus her ability to reason using the knowledge to connect source domain items that do not appear in an existing source to target mapping to items that do appear in a mapping. However, to make the answer more explicit, there are a number of issues that need to be addressed.

\subsection{PretenceSpaces}

Lakoff states that the reader can reason within the terms of the source domain. But what does this mean, and where does this reasoning take place? After all, our normal, common sense knowledge should tell us that there is no such location as 'the fast lane on the freeway of love' and so an explicit set of reasoning rules may well disallow the inference that the journey is exciting. It might be objected that using knowledge that there is no such location as 'the freeway of love' is somehow excluded because reasoning takes must place "within the terms of the source domain", and 
the knowledge that 'freeways of love' do not literally exist does not fall within the terms of the source domain. Consequently, such knowledge should not be used in the reasoning. We believe that this is broadly correct, but needs further specification. However, consider the source domain of journeys. Excitement at fast travel may arise because fast travel is dangerous and a degree of danger is exciting. But if we are talking of danger causing excitement, then we might question whether we are still reasoning within the (source) domain of journeys or whether elements of other domains are also being used. In fact, we are somewhat sceptical of the utility of "domain" as an explanatory principle and will use the term in this paper merely as a useful means of describing the source subject matter.

What is needed is some way to protect the within-source reasoning from being falsified by our knowledge that the objects being referred to in the source do not literally exist, or that propositions holding in the source are not literally true. We shall introduce the term 'pretence cocoon' or 'pretence space' 3 to describe where this protected reasoning about the source can take place. In the pretence cocoon reasoning proceeds on the assumption that the metaphorical utterance is literally true and we might think of propositions inside the pretence cocoon as forming a description of an imaginary, pretend world. However, apart from this constraint, in principle, knowledge from any putative domain may be used in the reasoning process. Thus knowledge about danger and excitement as well as journeys may be used. Outside of the pretence space propositions are about reality ${ }^{4}$ as the reader sees it, and will in particular be about the target domain of the metaphorical utterance in question. The metaphorical mapping links hold between the inside of the pretence cocoon and the outside.

For the freeway-of-love example, the reader pretends, within the cocoon, that the lovers really are driving along a freeway. Consequences of this, using knowledge about freeway driving, can be worked out in the pretence cocoon, and some consequences may then be able to be transformed, via metaphorical mapping links, to become propositions about the love relationship that are outside.

\footnotetext{
This pretence space can be seen as loosely related to the blend space in Blending Theory, (Fauconnier and Turner 1998).

4 This is a slight simplification, since in mixed metaphors, one pretence space may be nested inside another pretence space, (Lee and Barnden 2001a).
} 


\subsection{Ancillary Assumptions}

Another aspect of Lakoffs proposal that needs further clarification is the issue of exactly what information is contained within a conceptual metaphor. With our stress on the importance of pretence cocoon inferencing for metaphor understanding, we are now doing more than just identifying which source to target links from a particular conceptual metaphor match the objects in the source, and then transferring the information out to reality. The question then arises as to whether the specification of a conceptual metaphor should consist solely of a list of mapping links. If we are to reason about travellers and their particular journey, etc, then we ought also to assume that the lovers/travellers are alone on their journey in order for a number of transferable inferences about the love relationship to be made. Consequently, if we are to give a full specification of the conceptual metaphor, then we need to include along with a list of mapping links, some 'ancillary assumptions' that do not map onto any target domain equivalent, but are just about the source domain and allow certain source domain inferences to be made.

\subsection{Additional Mappings}

Finally Lakoff s proposal needs further clarification on the issue of where the source to target mapping links are to be found. We shall shortly examine instances where after a process of inferencing a connection is made to an element that would generally be accepted to form a part of a conventional metaphor.

Grady (1997) has persuasively argued that many putative conceptual metaphors should be reanalysed as combinations of a number of more basic conceptual metaphors, or 'primary metaphors'. However, we would not wish to claim that the excitement to excitement mapping is a very basic metaphor that combines with others to form, in this instance, the LOVE IS A JOURNEY conceptual metaphor; the invariant mapping from excitement to excitement is not a metaphor. Instead, we shall draw upon a proposal of Carbonell (1981) that certain aspects of sources are invariant and tend to map over to targets, irrespective of the particular conceptual metaphor in use. We claim that these aspects of the source are adjuncts to the mappings specified in conceptual metaphors. That is they are 
'conceptual metaphor mapping adjuncts' or COMMAs ${ }^{5}$. By analogy with 'comma intonation ${ }^{6}$, these COMMA mappings add additional mappings to the main mappings. As we shall see, in many metaphors the conceptual metaphor merely acts as a place-holder for the COMMA and the main information being conveyed by the metaphorical utterance is actually provided by the COMMA. Furthermore, we claim that they can be partitioned into a number of different types, at least as a first step, although, we do not rule out the possibility that some more fundamental principle underlies all of them. Thus, the mapping from excitement to excitement, we would claim, is an instance of the 'mental/emotional states COMMA'.

Mental/Emotional States COMMA:

If some agents in the source domain have correspondents in the target domain that are also agents, then their mental and emotional states map identically, except that the objects or propositional contents (if any) of the states are modified suitably by any mapping relationships that apply, and provided that this modification can be done. E.g., if John and Mary arguing is metaphorically viewed as their engaging in physical combat, then the source domain proposition that John believes he is losing in the combat maps to the target domain proposition that John believes he is losing the argument assuming that combat-losing maps to argument-losing. The COMMA allows for object/content-free states. Thus, John's being excited in the source domain maps to John's being excited in the target domain.

This COMMA, and indeed all COMMAs, are merely defaults, that is they may be overridden by specific target domain information to the contrary. In section 3, we shall look at more examples of COMMAs, and present an initial, and no doubt partial, list of different COMMA types. However, since we claim that the act of inferencing within the terms of the source domain involves a complex interaction between (possibly many different) COMMAs and conventional source-to-target links, we shall now look at an example where the process of reasoning connects map-

\footnotetext{
5 In other papers, (e.g., Barnden and Lee 2001a, Barnden et al 2003) we prefer the terms 'Metaphorical View' to 'Conceptual Metaphor', and 'View Neutral Mapping Adjuncts' or VNMAs to COMMAs. Apart from wishing to remain agnostic on many of the claims being made about conceptual metaphors, we would not wish to claim that COMMAs apply only to conceptual metaphors. They may, for instance, also apply to image metaphors.

6 Comma intonation is the intonation contour typically assigned to phrases in apposition, parentheticals, non-restrictive relative clauses, and so on. In other words, phrases that are not part of the main clause, but which add information to it. Although they are not part of the main clause, they may provide much of the main informational contribution of the sentence, e.g. my colleague, who has been researching temporal metaphors, was there.
} 
transcending elements to links that are usually thought to be part of a conceptual metaphor as well as to a COMMA.

\subsection{Inferencing in Metaphor Comprehension: The Far-Reaches of one's Mind}

In the far reaches of her mind, Anne knew Kyle was having an affair, but "to acknowledge the betrayal would mean I'd have to take a stand. I'd never be able to go back to what I was familiar with," she says. Not until eight months had passed and she finally checked the phone bill did Anne confront the reality of her husband's deception. In Linden Gross, "Facing up to the Dreadful Dangers of Denial," Cosmopolitan, 216( 3), USA ed., March 1994. Bold font added.

Consider the clause we have depicted in bold. Let us assume that there isn't already an established mapping from the 'far reaches' of a physical space into some aspect of the mental domain. The principle of 'MapExtension Minimization obliges us to give only a very low priority to creating such a mapping for 'far reaches'. A further principle, that of 'TransferWarranting by Unmapped Structure' requires that the existence of unmapped structure is not ignored, but forces an 'on-the-fly' reasoning process in the source domain to attempt to inferentially link the term 'far reaches' to items for which there is an existing mapping.

Transfer-Warranting by Unmapped Structure.

In the case of map-transcending metaphorical utterances, the warrant for attempting to transfer a source-domain representation may include inferential linkage to [A] complex source-domain structures that arise from the utterances at hand and that are, typically, not themselves mapped to parallel structures in the target domain, and $[\mathrm{B}]$ general knowledge of the source domain that, again, is typically not mapped to the target.

For this example,we assume that there are two relevant conceptual metaphors: MIND IS PHYSICAL SPACE and IDEAS ARE PHYSICAL OBJECTS. The former contains the following relevant mapping:

When a person's mind is being viewed as a physical space, an idea's being physically located in the space corresponds to the person's being able to operate mentally on the idea, to a very low degree at least.

We also assume that this conceptual metaphor must contain the following two ancillary assumptions that enrich the nature of the alleged physical space rather than forming source to target mappings: 
When a person's mind is being viewed as a physical space, the person's conscious self is viewed as a person physically located in that space. Moreover, if the space has a main sub-region, the conscious self is in that sub-region.

When an agent $\mathrm{X}$ is viewed as having a mental state or process physically within some sub-region $\mathrm{L}$ of his/her mind, then the ideas involved in that state or process are normally also physically located in L.

The second ancillary assumption reflects the fact that in uses of MIND IS PHYSICAL SPACE in real discourse, mental states and processes are often portrayed as having physical locations in the mind.

As for IDEAS ARE PHYSICAL OBJECTS, the only mapping the reader needs to possess is as follows:

When an idea entertained by a person is being viewed as physical object, and the person's conscious self is viewed as a person, then the ability of the conscious self to physically operate on the idea corresponds to the real person's ability to operate in a conscious mental way on the idea.

We suggest that a likely informational contribution of the bold clause to the understanding of the discourse as a whole is the proposition that

The idea that Kyle was having an affair was something of which Anne had only a very low degree of conscious awareness (i. e. on which Anne had only a very low ability to mentally operate in a conscious way).

A simulation of the reasoning involved in this example has been performed on our computer system, ATT-Meta, a computer system which performs, among other things, reasoning about information derived from metaphorical utterances, (Barnden and Lee 1999, Barnden and Lee 2001a, Barnden et al 2002). Informally, the contribution can be produced as follows.

We can gloss the direct source-domain meaning of the clause as follows:

physically-in the far reaches of Anne's mind, Anne knew that Kyle was having an affair.

That is, Anne's 'knowing' state is located in a specific sub-region of her mind, namely the 'far reaches' sub-region. The second ancillary assumption states that when a mental state such as this 'knowing' mental state is located within a sub-region, then the ideas involved in that state, i.e., the idea that Kyle was having an affair, are also located in that subregion. Consequently, the reader can infer within the pretence cocoon that 
the idea $\mathrm{K}$ that Kyle was having an affair was in the far reaches of Anne's $\operatorname{mind}^{7}$.

The first ancillary assumption locates Anne's conscious self within the main sub-region of her mind. We can assume that the reader has the everyday general knowledge that if something is far away, it is hard to operate on it. Consequently, the distance between the far sub-region and the central sub-region in which Anne's conscious self is located entails that the conscious self had only a very low level of ability to operate physically on the idea.

The mapping link from the IDEAS ARE PHYSICAL OBJECTS conceptual metaphor can now be used to transfer this physical operability to a mental operability.

This doesn't quite give us the informational contribution of the bold clause to the understanding of the discourse. Strictly speaking what the conceptual metaphor provides is a mapping from physical to mental operability. The IDEAS ARE PHYSICAL OBJECTS conceptual metaphor says nothing about a correspondence between the degree of physical and mental operability. Consequently, on its own, it would not distinguish between the sentence under consideration and a sentence such as 'Anne had an idea in mind'. What we need to ensure is that only a very low degree of operability maps from the physical to the mental domain. We could rewrite this conceptual metaphor to include correspondences between degrees of operability but we believe this would be a mistake. Thus consider the following two sentences, which were found doing an internet search:

"He's only dimly aware of the reason for his placement in the symbolic order"

"Now you've almost reached your destination: an informed and thoughtful opinion."

The former involves the Conceptual Metaphor UNDERSTANDING AS SEEING, but by using the word 'dimly', it is suggested that the individual has only a low degree of awareness or understanding of the reason for his placement. Similarly, the latter involves the Conceptual Metaphor PURPOSEFUL ACTION AS A JOURNEY, with the degree carried by 'almost

\footnotetext{
If we were to be completely explicit then we would require a means of getting this information into the pretence cocoon. In the ATT-Meta system, this is achieved via a target to source transfer rule.
} 
reached'. In short, adding something like a degree variable to various separate mappings would be to miss the significant generalization that the degree to which something holds is an important part of the informational contribution of numerous conceptual metaphors. Furthermore, we have already discussed a mechanism to achieve such a general cross-domain mapping. What is needed is another COMMA; in this case, a Qualitative Degree COMMA.

If the holding of a graded property or relationship in the source maps to the holding of a graded property or relationship in the target, then the qualitative absolute and relative degrees map over identically. For example, if presence of above-normal temperature maps to presence of anger, then a high temperature maps to intense anger, and the higher the temperature the more intense the anger.

We shall take a further look at COMMAs in the next section and try to motivate some more examples. However, we shall first make a few concluding comments about the current example.

\subsection{Discussion}

The above example makes a very strong case for a principle such as "Map-Extension Minimization". Suppose that understanding the clause actually does involve an allegation that Anne's mind has some aspect corresponding to the 'far reaches' of the physical space. But what could it be? Finding such a correspondent would probably be impossible for an ordinary reader, because there is no generally held, non-metaphorical, common sense or scientific model of mind that would allow the identification of an aspect that would count as "far reaches." However, if we use COMMAs, there no need to map 'far-reaches', or more generally to map relationships of physical distance within the mind-space to mental relationships within the mind. Rather, it is a consequence of physical distance, namely a very low degree of physical operability for the idea in question, that ends up being mapped to the target domain.

It is worth contrasting the above account with the approach briefly sketched and rejected in the Introduction, but which, nonetheless, is quite widely assumed in much metaphor research e.g. in approaches based on analogy systems such as SME (cf.: Falkenhainer et al. 1989), ACME (cf.: Holyoak, \& Thagard, 1989) and IAM (c.f.: Keane 1988). In these 
approaches, information in the source domain is transferred if there is a detailed structural mapping between other aspects of the source and target domains, and if the source domain information for which no mapping is yet established is connected to these aspects of the source domain which are mapped. Our account shows, via the reasoning process, the connectivity of the map-transcending elements to elements that do map, but crucially does not attempt to make a mapping for the relations/connections so uncovered.

To conclude, we postulate that the following sub-processes are involved in the process of understanding map-transcending utterances:

- construction of the direct source-domain meaning of the utterance

- placing of it in the pretence cocoon

- usually, performance of (predominantly) source-domain reasoning within the pretence cocoon, using that direct meaning together with general knowledge about the source domain

- possibly, application of ancillary assumptions to create propositions within the pretence cocoon

- mapping acts, including at least one that goes from source to target; the acts can include applications of conceptual metaphor mapping adjuncts or COMMAs.

\section{Conceptual Metaphor Mapping Adjuncts (COMMAs)}

We have suggested that it is often the case that many of the source to target mapping links used in transferring features, relations, propositions, etc from a source to a target do not belong to one of the established conceptual metaphors; and, nor are the mappings created on-the-fly. Instead, they belong to mapping relationships (or schemes for such relationships) that apply, by default ${ }^{8}$, whatever particular conceptual metaphor is at hand. They are parasitic on other things that are mapped, in the sense that they are all of the form: if such-and-such things are mapped, then also such-and-such things are mapped.

\footnotetext{
8 As stated in section 2.4, COMMAs are defaults; they will apply unless there is specific target domain information to the contrary.
} 


\subsection{Identifying COMMAs}

So far we have argued for two, quite different, COMMAs: Mentall Emotional State and Qualitative Degree. What further COMMAs are required is very much the topic of on-going research. COMMAs may be found by first considering what the likely meaning and connotations of a metaphorical utterance might be and then carefully examining whether any proposed conceptual metaphors or sets of mappings fully account for the meaning. This process can expose gaps and some of these may be filled by the type of invariant mapping from source to target we have discussed. If similar invariant mappings are found to be required across a range of other conceptual metaphors, then we can capture this significant generalization by treating the mapping as another COMMA. For example, the time order of events in the source appears almost invariably to be replicated in the target, yet the need for this invariant mapping of time-order is almost never remarked upon.

However, there is a further source of COMMAs and that is by examining existing mappings, not for lacunae, but to determine whether they incorporate invariant aspects. The existence of COMMAs can expose missing generalizations. For example, in section 2.5, we considered how to capture the information that only a very low degree of manipulation is afforded by a distant object and dismissed the option of having a range of mappings. A range of mappings would include one for the instance where only a very low degree of manipulation is possible, one for a high degree and so on. We also dismissed the option of specifically adding a degree variable to the IDEAS-AS-PHYSICAL-OBJECTS conceptual metaphor. Pursuing either of these options, we argued, would be to miss the generalization that many other metaphors, which are not examples of IDEAS-AS-PHYSICALOBJECTS, also involve the invariant transfer of degrees. However, a careful examination of some of the mappings proposed in the literature can reveal what are essentially invariant mappings between source and target and many of these might better be treated as COMMAs. Recall, from section 2.1, the mappings that Lakoff suggested comprised the LOVE IS A JOURNEY conceptual metaphor:

The lovers - $>$ travellers.

The love relationship - $>$ the vehicle.

Common goals $->$ common destinations.

Difficulties in the relationship - > impediments to travel. 
Under our proposed approach whenever the left-hand-side of a mapping includes material corresponding broadly to the right-hand-side, we have a possible candidate for an invariant property that can be factored out of the specific mapping. So, let us consider the final mapping (relationship difficulties-impediments to travel), and an example of the type of metaphorical utterance that this mapping is intended to account for: ' $i t$ 's been a long bumpy road'. This looks like a plausible candidate. As written, the righthand-side of the mapping involves a journey and the left-hand-side, a relationship but this seems unnecessarily restrictive since we can infer from the use of the word 'road' that the source domain concerns a journey. So, let us omit the fourth mapping. This reduces the number of separate mappings, giving a more economical account if we can somehow account for the effect of the difficulties-impediments mapping,

A consequence of this omission is that the 'bumps' must be considered what we are calling a Map Transcending Entity and thus must initiate a sequence of inference steps via the principle of Transfer-Warranting by Unmapped Structure ${ }^{9}$. One important step in the process is the inference that bumps in the road cause the journey not to proceed properly. On the basis of other examples in which causal relationships appear to map in an invariant manner, we have proposed a CAUSATION COMMA. This general COMMA, in conjunction with the remaining specific mapping from the LOVE IS A JOURNEY conceptual metaphor, accounts for the effect of Lakoffs fourth mapping. Note, it might further be inferred that bumps are uncomfortable, causing emotional discomfort, and so subject to our EMOTIONAL-STATES VNMA. Thus we get a richer reading than if we had used Lakoff s mapping.

Finally note that the COMMAs stipulate source to target mappings that are typically not included amongst the source to target mappings of the conceptual metaphors. There is no assumption that these mappings must play a major or a minor role in the informational contribution of metaphorical utterances to discourse. Whether they do or not depends on the particular conceptual metaphor, the particular metaphorical utterance and the discourse in which the metaphor is embedded.

\footnotetext{
9 Note that 'bumps' do not actually represent the entity on the left-hand-side of the mapping and so should strictly be considered as a MTE even with the presence of the fourth mapping. However, far less inferencing would be required.
} 


\subsection{Current COMMAs}

The following list is based on that of Barnden \& Lee ${ }^{10}$ (2001a), but is slightly simplified. Note that we refer to target domain items to which source domain items are mapped as 'mappees'.

Causation/Ability COMMA: These COMMA mappings cover a range of related relationships that hold between events or other entities. They include: causation, prevention, helping, ability, (dis)enablement and function/purpose. Causation is the most prototypical of these relations. So, if there is a relationship between A and B in the source, such that A causes $\mathrm{B}$, and if $\mathrm{A}$ maps onto $\mathrm{P}$ and $\mathrm{B}$ maps onto $\mathrm{Q}$ where $\mathrm{P}$ and $\mathrm{Q}$ are in the target, then by default, there is also a causal relationship between $\mathrm{P}$ and $\mathrm{Q}$ in the target domain. Note that it is the existence of the relation between $A$ and $B$ that maps over, not the specifics of how $A$ causes $B$. If $A$ causes $B$ not to happen, then we can say that A prevents B. If the presence/ existence of $A$ (dis)enables $B$ to happen, then $A$ (dis)enables $B$. A's causing of $B$ does not entail that $B$ is the intended consequence of $A$. If $B$ is the intended consequence of $\mathrm{A}$, then we can describe the function/purpose of $\mathrm{A}$ as $\mathrm{B}$. Thus turning the key in the ignition causes the car to start. Since the car starting is the intended consequence of turning the key, we can say that the purpose behind turning the key is to start the car.

Mental/Emotional States COMMA: These COMMA mappings also cover a range of related relationships. Their description was given earlier.

Value-Judgment COMMA: Levels of goodness, importance or other types of value assigned by the understander to states of affairs in the source domain map identically to levels of goodness, etc., of their mappee states of affairs, if any. So, for example, in the phrase, 'that's a gem of an idea', we can assume that gems are particularly esteemed, valuable, etc. These value judgements, then, transfer over to the mappee.

Time-order COMMA: The time-order of events in a source domain is the same as that of their mappee events, if any.

Duration COMMA: Qualitative length of time, in the context of the source domain, that is consumed by an event maps identically to qualitative

10 In this paper COMMAs are termed VNMAs (View Neutral Mapping Adjuncts). 
length of time, in the context of the target domain, consumed by the mappee event, if any. E.g., if something takes a long time in the context of the source domain then its mappee takes a long time in the context of the target. Also, qualitative duration comparisons map over.

Rate COMMA: Qualitative rate of progress of an event in the source domain maps identically to qualitative rate of progress of its mappee, if any. E.g., if an event progresses slowly in the context of the source domain, then its mappee progresses slowly in the context of the target domain.

Event-Shape COMMA: Aspectual features of events, such as whether they have a start or end, or are intermittent, map identically to any mappee events.

Uncertainty COMMA: The level of certainty with which situations hold in the source maps at least roughly to level of certainty with which their mappee situations, if any, hold.

Modality COMMA: Relative degree of necessity, possibility, obligation, tendency, etc. in the source domain, for actors to undertake actions or for a state of affairs to obtain, maps identically to relative degree of necessity, possibility, etc., in the mappee situations, if any.

Qualitative Degree COMMA. The description of this COMMA was given earlier.

Set-Size COMMA: Qualitative size (relative or absolute) of sets in the source domain maps identically to qualitative size of mappee sets in the target domain. E.g., if a set is large in the terms of the source domain then its mappee set (if any) is large in the terms of the target domain.

Logical structure COMMA: A special case of this COMMA is negation: If a property or relationship in the source has a mappee property or relationship, then non-possession of the source property/relationship maps to non-possession of the target property/relationship. Other logical structures such as conjunction and disjunction are similarly mapped.

Note that for some COMMAs, it is often the case that a degree of source domain reasoning is required in order to connect to a source to target mapping link. However, we would not claim that this is true all the time and for all COMMAs. For example, little reasoning is probably required 
to determine the time order of events in the source domain. When this is determined it is mapped over to the target domain via the Time-order COMMA. As we have suggested, it is also often the case that the COMMA mappings make a substantial input to the informational contribution of the metaphorical utterance. However, again, this is not always the case, and for some COMMAs such as Time-order, it may rarely be the case.

We shall now examine a number of metaphorical utterances that require some of these COMMAs. All of the examples are real. The first two were taken by Goatly (1997) from a corpus and are discussed along with others from Goatly (1997) in Barnden (2001).

\subsection{COMMAs in Use}

\subsubsection{The Battered Trilby}

And towards the end of the century men began to wear, so to speak, the very symbol of their bashed-in authority: the trilby. [Goatly p.127, bold font and hyphens inserted by us; from corpus]

The focus of Goatly's discussion is the metaphor (as he claims) of AUTHORITY AS TRILBY HAT ${ }^{11}$. However, for brevity we will ignore the trilby and discuss merely the phrase "bashed-in authority" on the simplifying pretence that a hat is not mentioned at all. We can take this phrase to manifest a familiar, very general conceptual metaphor of ABSTRACT ENTITY IS PHYSICAL OBJECT. We assume that 'the bashed in authority' is placed in the pretence cocoon, where it is treated as a physical object and in particular as something that can actually be 'bashed in'. Prototypically, bashed-in things are rigid walls, doors, etc: perhaps exterior physical surfaces in general. As a result of being bashed in, such a physical object is damaged, i.e., it is not as it should be and probably lacks to some extent the ability to perform its function. Thus, we might describe the informational contribution of the phrase in bold to the rest of the discourse as:

The authority is probably partially defunct and is not as it should be.

The metaphor is map-transcending because there is no need for the specific physical notion of bashing-in to be mapped to the target domain.

11 Capitalised by us. 
Instead, the reader can infer within the pretence cocoon that if something is bashed in then it is probably defunct to some extent. The reader can also infer that if something is said to be bashed in, then the person making the claim has negative feelings about the object; it is not as it should be. These relations/attributes involving the authority can be transferred to reality via the Causation/Ability and the Value-Judgment COMMA mappings respectively. Finally, note that the informational contribution of the phrase is largely carried by these two COMMAs.

\subsubsection{The Neck-Cricked Managers}

This all means that general managers have cricks in their necks from talking down to the Community Health Councils and District Health Authorities, and up to Regions and the Department. [Goatly p.162; from Daily Telegraph newspaper]

This manifests the familiar conceptual metaphor ORGANIZATIONAL CONTROL IS VERTICAL POSITION. For simplicity we will ignore the possibility that "talking down to" might be taken as having its stock metaphorical meaning of "being condescending to." Notice also that talking is an aspect both of the source domain and the target domain.

In the pretence cocoon, the managers get cricks in their necks because of their contortions. The managers therefore experience physical suffering, and hence negative emotions about the cause of that suffering. The causation and negative emotions map to the target by Causation/Ability and Mental/ Emotional States COMMAs. The latter maps the mental/emotional states of source-domain agents about source-domain aspects over to mental/ emotional states of corresponding target-domain agents, (a more refined statement is given in Barnden \& Lee (2001a).

It could further be inferred from the utterance that the managers have some inability to continue talking easily to their controllers and controllees. This would follow from inferring within the pretence cocoon that the cricks cause some inability to turn heads, so that there is difficulty in talking, and then applying COMMAs. The Causation/Ability COMMA maps the causation and the inability to the target domain. Also, the degree of inability is mapped by the Qualitative Degree COMMA.

There is no need to find a mapping relationship that handles necks, cricks, or head-turning themselves. Thus these elements are map- 
transcending. Finally, note that again, the principal information contribution of the utterance is conveyed by the COMMAs

\subsubsection{Mind Is A Physical Object}

It was only momentum that kept Alice's gears of thought and analysis grinding past any practical application. Jane Smiley, Duplicate Keys, p.206. London: Flamingo (Harper Collins), 1996. Popular novel.

This manifests the familiar conceptual metaphor MIND IS A MACHINE, which is a special case of MIND IS A PHYSICAL OBJECT. We assume that one of the main informational contributions, arising from 'momentum', is that Alice's thinking activity up to the relevant point in time caused her to continue thinking (without practical application). This contribution arises under our approach as follows.

Source-domain reasoning leads to the conclusion that Alice's mindmachine kept on going but against the resistance of the gear-grinding. The cause of this was the 'momentum'. This implies that the machine's running up to this point is the cause of the continued running. We assume that, under the metaphorical view, operation of the machine maps to active thinking. Then with the aid of the Event-Shape and Causation/Ability COMMAs we get the mentioned informational contribution.

Also, the gear-grinding suggests in the source domain that the machine is tending to stop. This tendency maps to a tendency of Alice's mind to stop its thinking activity, because of the Modality and Event-Shape COMMAs.

\section{Discussion}

We have argued in this paper that (a) for many non-stock metaphors, it is incorrect to assume that all the elements of the source domain map to elements of the target domain, and (b) that a much more economical set of mappings should be used instead. Given the extreme unlikelihood that all but a few of the notions in the source domain of any metaphorical utterance would match explicit source to target mapping links, we believe that we have no other choice. However, it does force us to consider explicitly how to treat these map-transcending elements. Our answer is to emphasise the 
role of reasoning using these source domain elements, which we argue takes place in a special space: the pretence cocoon. Here, the metaphor is taken to be literally true, and an attempt is made to connect the maptranscending elements to any mapping links coming from conceptual metaphors or from COMMAs, and possibly employing ancillary assumptions from a conceptual metaphor in the process.

Note that our search for COMMAs should be thought of as complementary to the somewhat similar endeavour undertaken by Grady (1997a\&b), who looks for recurring primary metaphors in proposed conceptual metaphors. As we have said earlier, these are very different from our invariant mappings, which one would not wish to call metaphors. Nonetheless, the view that much of the ontological richness of contemporary conceptual metaphors should be factored into more basic mappings which can then combine and interact with each other to give the results is a view we share.

We might also note that we can account for certain mapping gaps in the sense of Grady. Let us return to the discussion in section 2.5, where we argued that sentences such as the idea lay in the far reaches of her mind can be understood by assuming a MIND AS PHYSICAL SPACE conceptual metaphor, an IDEAS AS PHYSICAL OBJECTS conceptual metaphor, the DEGREE COMMA, and the inference that a distant object can only be manipulated to a limited degree. Suppose we tried an alternative spatial term:

$\#^{12}$ Anne had an idea on the right of her mind.

According to Kilgarriffs (2003) analysis of the BNC, 'right' is the most frequent spatial term in the $\mathrm{BNC}$ and yet this sentence seems to be extremely odd. Why should this be? Consider what might license the use of the term here? Unlike the 'far reaches' example, objects on the right are not in a distant location, presenting a challenge for easy manipulation. Thus the DEGREE VNMA cannot license the term. It is just possible that in an appropriate context, the term might be used to convey a value judgment about the idea, but there is no context here. In short, if there is no mapping or COMMA to license and so ultimately ground the inference process initiated by the existence of a map-transcending entity such as 'the right of her mind', then Transfer-Warranting by Unmapped Structure will fail to apply.

12 We will use a '\#' to indicate a sentence which is hard to interpret. 
We have suggested that for many metaphors the conceptual metaphor merely acts as a place-holder for the COMMA and that the main information being conveyed by the metaphorical utterance is provided by the COMMA. This goes some way towards explaining certain problems that have been noted with some exemplars of conceptual metaphors. Describing a particular metaphor as an example of a specific conceptual metaphor may appear somewhat forced if we have the assumption that the main informational contribution of the metaphor has to do with the mappings described by the conceptual metaphor. Consider 'our love is a roller coaster ride'. Lakoff analyses this as an example of LOVE IS A JOURNEY. Glucksberg and McGlone (1999) argue that people recognize 'a roller coaster ride' as an exemplar of a class of exciting experiences. We might suggest that this is an example of the conceptual metaphor LOVE IS A JOURNEY, but where the main import of the utterance is carried by the emotional states COMMA, and this is achieved via the reasoning that "a roller coaster ride" is an exciting experience.

Note however, that the approach outlined in this paper does have some parallels with Glucksberg's 'Categorization' approach. Our assumption that only a minimal set of central mappings are required to understand a complex source domain containing elements (MTEs) that do not find direct correspondences in the target mean that a small number of mappings can explain a seemingly wide variety of related metaphorical utterances. It could loosely be described, therefore, as a form of categorization approach as opposed to a direct comparison approach in which source domain structure would map point-by-point onto target domain structure.

The existence of COMMAs allow metaphorical expressions to be characterized along a number of different dimensions, and part of the task of the metaphor analyst is to determine what these dimensions are and how they interact. There will, of course, be the dimension of the main conceptual metaphor, but much, at least, of the flavour of the metaphorical utterance is likely to be carried by one or more COMMAs. Determining what these are, how they interact with both the main conceptual metaphor, with each other, and with any map-transcending elements is no trivial matter.

Although we have barely mentioned our computational implementation of aspects of the approach outlined here, we should stress that our approach 
to metaphor analysis has gone hand in hand with an attempt to implement our analysis on a computer. This has forced us to address issues that are all too frequently overlooked such as the need for ancillary assumptions, or the need to protect the source domain reasoning from conflicting evidence in reality, or even the need for COMMAs. In short, we believe that a computational account, or at least an account of metaphor given in algorithmic detail, is desirable. We hope we have shown that such an account does not detract from an understanding of the nature of metaphor in all its richness, but is a crucial tool in the process of uncovering these riches.

E-mails: A.M.Wallington@cs.bham.ac.uk J.A.Barnden@cs.bham.ac.uk S.R.Glasbey@cs.bham.ac.uk M.G.Lee@cs.bham.ac.uk

\section{REFERENCES}

Barnden, J.A. 2001. Application of the ATT-Meta metaphorunderstanding approach to selected examples from Goatly. Technical Report CSRP-01-01, School of Computer Science, University of Birmingham, UK.

, Glasbey, S.R., Lee, M.G. \& W Allington, A.M. (2004), Varieties and directions of inter-domain influence in metaphor. Metaphor and Symbol 19.1.

\& M.G. LeE. 1999. An implemented context system that combines belief reasoning, metaphor-based reasoning and uncertainty handling. In P. Bouquet, P. Brezillon \& L. Serafini, Eds. Second International and Interdisciplinary Conference on Modeling and Using Context (Context '99), Lecture Notes in Artificial Intelligence, Vol. 1688: 28-41. Springer. 2001a. Understanding usages of conceptual metaphors: An approach and artificial intelligence system. Technical Report CSRP01-05, School of Computer Science, University of Birmingham, UK. . 2001b. Application of the ATT-Meta metaphor-understanding system to an example of the metaphorical view of MIND PARTS AS PERSONS. Technical Report CSRP-01-09, School of Computer Science, University of Birmingham, UK. 
Carbonell, J.G. 1981. Invariance hierarchies in Metaphor Interpretation. In Proceedings of.of the Third Annual Meeting of the Cognitive Science Society, August 1981, pp 292-295.

Falkenhainer, B., K.D. Forbus, \& D. Gentner. 1989. The StructureMapping Engine: algorithm and examples. Artificial Intelligence, 41 (1), 1-63.

Gentner, D, 1983. Structure Mapping: A Theoretical Framework for Analogy. Cognitive Science, 7 (2), pp 155-170.

Glucksberg, S \& M S. McGlone. 1999. When love is not a journey: What metaphors mean. Journal of Pragmatics, 31, 1541-1558.

Goatly, A. 1997. The language of metaphors. London and New York: Routledge.

Grady, J. E. 1997a. THEORIES ARE BUILDINGS revisited. Cognitive Linguistics, 8 (4), pp. 267- 290.

1997b. Foundations of meaning: Primary metaphors and primary scenes. UC Berkeley Dissertation, Dept. of Linguistics.

Holyoak, K.J. \& P. Thagard, P. 1989. Analogical mapping by constraint satisfaction. Cognitive Science, 13 (3), 295- 355.

Gross, L. 1994. Facing up to the Dreadful Dangers of Denial. Cosmopolitan, 216(3), USA ed.

INDURKYHA, B. 1987. Approximate semantic transference: A computational theory of metaphors and analogy. Cognitive Science, 11: 445-480.

Kilgarriff, A, 2003. BNC word frequency list. http:// www.itri.brighton.ac.uk/Adam.Kilgarriff/bnc-readme.html.

Keane, M.T. 1988. Analogical Problem Solving. Chichester: Ellis Horwood.

Lakoff, G. 1993. The contemporary theory of metaphor. In A. ORtony (Ed.), Metaphor and Thought, 2nd edition. Cambridge University Press. . 1994. What is metaphor? In J. A. BARnden \& K. J. HolYOAK (Eds.), Advances in Connectionist and Neural Computation Theory, Vol. 3: Analogy, Metaphor and Reminding, pp. 203-258. Norwood, N.J.: Ablex Publishing Corp.

\& M. Johnson. 1980. Metaphors we live by. Chicago: University of Chicago Press.

\& M. Turner. 1989. More than cool reason: a field guide to poetic metaphor. Chicago: University of Chicago Press.

Lee, M.G. \& J.A. Barnden. 2001a. Reasoning about mixed metaphors with an implemented AI system. Metaphor and Symbol, 16 (1\&2): 29-42. 
2001b. Mental metaphors from the Master Metaphor List: Empirical examples and the application of the ATT-Meta system. Technical Report CSRP-01-03, School of Computer Science, University of Birmingham, UK.

Ortony, A, D. Schallert, R. Reynolds, \& S. Antos. 1987. Interpreting metaphors and idioms: Some effects of context on comprehension. Journal of Verbal learning and Verbal Behaviour. 17:465-477

Smiley, Jane. 1996 Duplicate Keys, p.206. London: Flamingo, Harper Collins. VEALE, T. \& M.T. KEANE. 1997. The competence of sub-optimal structure mapping on 'hard' analogies. In Proceedings of the International Joint Conference on Artificial Intelligence. Nagoya, Japan. 